\title{
Promotion of compound K production in Saccharomyces cerevisiae by glycerol
}

\author{
Weihua Nan ${ }^{1}$, Fanglong Zhao ${ }^{1}$, Chuanbo Zhang ${ }^{1}$, Haiyan Ju ${ }^{1}$ and Wenyu Lu Lu $^{1,2,3^{*}}$ (D)
}

\begin{abstract}
Background: Ginsenoside compound K (CK), one of the primary active metabolites of protopanaxadiol-type ginsenosides, is produced by the intestinal flora that degrade ginseng saponins and exhibits diverse biological properties such as anticancer, anti-inflammatory, and anti-allergic properties. However, it is less abundant in plants. Therefore, enabling its commercialization by construction of a Saccharomyces cerevisiae cell factory is of considerable significance.

Results: We induced overexpression of PGM2, UGP1, and UGT1 genes in WLT-MVA5, and obtained a strain that produces ginsenoside CK. The production of CK at $96 \mathrm{~h}$ was $263.94 \pm 2.36 \mathrm{mg} / \mathrm{L}$, and the conversion rate from protopanaxadiol (PPD) to ginsenoside CK was $64.23 \pm 0.41 \%$. Additionally, it was observed that the addition of glycerol was beneficial to the synthesis of CK. When $20 \%$ glucose (C mol) in the YPD medium was replaced by the same $\mathrm{C} \mathrm{mol}$ glycerol, CK production increased to $384.52 \pm 15.23 \mathrm{mg} / \mathrm{L}$, which was $45.68 \%$ higher than that in YPD medium, and the PPD conversion rate increased to $77.37 \pm 3.37 \%$ as well. As we previously observed that ethanol is beneficial to the production of PPD, ethanol and glycerol were fed simultaneously in the 5-L bioreactor fed fermentation, and the CK levels reached $1.70 \pm 0.16 \mathrm{~g} / \mathrm{L}$.

Conclusions: In this study, we constructed an S. cerevisiae cell factory that efficiently produced ginsenoside CK. Glycerol effectively increased the glycosylation efficiency of PPD to ginsenoside CK, guiding higher carbon flow to the synthesis of ginsenosides and effectively improving CK production. CK production attained in a $5-\mathrm{L}$ bioreactor was $1.7 \mathrm{~g} / \mathrm{L}$ after simultaneous feeding of glycerol and ethanol.
\end{abstract}

Keywords: Compound K, Saccharomyces cerevisiae, Glycerol, Metabolic engineering

\section{Background}

Ginseng has been used in treatment of cardiovascular and cerebrovascular diseases, neurasthenia, and physical weakness, and ginsenosides are the primary ingredients in Ginseng that elicit these physiological activities [1]. Ginsenoside compound K (CK), the primary deglycosyl metabolite of ginsenosides produced under the action of intestinal flora and absorbed into the body, has been proven to elicit anticancer, anti-inflammatory,

*Correspondence: wenyulu@tju.edu.cn

${ }^{1}$ School of Chemical Engineering and Technology, Tianjin University, Tianjin 300350, People's Republic of China

Full list of author information is available at the end of the article anti-allergic, antidiabetic, anti-angiogenic, anti-aging, neuroprotective, and liver protective properties among several notable biological characteristics [2]. Ginsenoside $\mathrm{CK}$ is a rare ginsenoside derived from ginseng saponins $\mathrm{Rb} 1$ and $\mathrm{Rb} 2$ by intestinal bacteria [3]. The method of synthesis of CK has garnered significant attention. Several studies have focused on the hydrolysis of major ginsenosides to active secondary ginsenosides using mild acid hydrolysis alkali treatment, microbial conversion, and enzymatic conversion [4-7].

Synthetic biological studies have seen persistent development in recent years, enabling microbial synthesis of compounds derived from animals and plants. For example, artemisinin is produced by Saccharomyces cerevisiae

(c) The Author(s) 2020. This article is licensed under a Creative Commons Attribution 4.0 International License, which permits use, sharing, adaptation, distribution and reproduction in any medium or format, as long as you give appropriate credit to the original author(s) and the source, provide a link to the Creative Commons licence, and indicate if changes were made. The images or other third party material in this article are included in the article's Creative Commons licence, unless indicated otherwise in a credit line to the material. If material is not included in the article's Creative Commons licence and your intended use is not permitted by statutory regulation or exceeds the permitted use, you will need to obtain permission directly from the copyright holder. To view a copy of this licence, visit http://creativeco mmons.org/licenses/by/4.0/. The Creative Commons Public Domain Dedication waiver (http://creativecommons.org/publicdomain/ zero/1.0/) applies to the data made available in this article, unless otherwise stated in a credit line to the data. 
[8], phloroglucinol is produced by Escherichia coli [9], pinene is produced by Corynebacterium glutamicum [10] and so on. $\mathrm{CK}$, a rare ginsenoside eliciting multiple biological activities, and can be synthesized using synthetic biological techniques as well. Zhou et al. synthesized $1.4 \mathrm{mg} / \mathrm{L}$ of CK using yeast [11]. Gwak et al. synthesized CK using tobacco as the host, and the concentration of CK achieved was 1.55-2.64 $\mu \mathrm{g} / \mathrm{g}$ CDW [12]. Li et al. overexpressed the key genes of the mevalonate (MVA) pathway and the fusion of PPDS-ARR1 in Yarrowia lipolytica, and the CK production in 5 - $\mathrm{L}$ bioreactor was $161.8 \mathrm{mg} / \mathrm{L}$ with fermentation optimization [13].

S. cerevisiae is a simple eukaryotic cell with a complete and mature eukaryotic expression system. Compared to the prokaryotic expression system, the complete organelle structure is beneficial to the further processing of proteins, thus ensuring the activity of enzymes. S. cerevisiae has a distinct genetic background and is biologically safe. S. cerevisiae is an important industrial host for the production of enzymes, drugs, and nutrients, as well as commercial chemicals and biofuels [14]. In CK synthesis, the glycosylation of PPD requires the participation of uridine diphosphate-glucose (UDPG). The supply of UDPG to yeast plays a significant role in glucoside biosynthesis [15]. Wang et al. overexpressed the gene PGM2 and UGP1 genes encoding phosphoglucomutase 2 and UTPglucose-1-phosphate uridylyltransferase 1, respectively, in S. cerevisiae that are involved in the glucose synthesis system of uridine diphosphate. The conversion rate of scutellarein into its glucoside increased from 75 to $92 \%$ [16].

Glycerol has been established as a low cost waste product due to the global production of biodiesel [17]. Thus, new markets or novel applications of glycerol, such as conversion into valuable products, need to be explored [18]. Fermentation of glycerol by Anaerobiospirillum has been shown to produce succinic acid, while citric acid has been produced by Yarrowia lipolytica, 1,3-propanediol, acetic acid, and butyric acid by Clostridium butyricum, and 3-hydroxypropionic acid by Klebsiella pneumoniae [19].

Apart from its low cost, glycerol has several other advantages. Glycerol undergoes greater reduction than glucose, as its metabolism generates higher quantity of $\mathrm{NAD}(\mathrm{P}) \mathrm{H}[20]$. Glycerol is metabolized through respiration manner in most microorganisms, including $S$. cerevisiae, and this implies that the use of glycerol does not exert the Crabtree effect in S. cerevisiae [21]. Glycerol can improve the stability of cell membrane proteins [22] and promote correct folding of nascent polypeptides [23]. Ginsenoside CK is a product of glycosylation of protopanaxadiol (PPD), and its production requires the participation of UDPG. Dutra et al. indicated that glycerol increases UDPG pyrophosphatase activity relative to glucose [24]. Wilson et al. observed that when S. cerevisiae is cultured in glycerol, the glycogen accumulated is more than that accumulated when it is cultured in glucose, and the precursor of glycogen synthesis is UDPG [25].

We constructed an S. cerevisiae strain WLT-MVA5 [26] that could produce PPD, and this strain is used as a parent strain for further engineering. The metabolic pathway is presented in Fig. 1. We overexpressed the genes associated with UDPG synthesis. Based on these advantages of glycerol, this study used glycerol as a partial carbon source and effectively increased the conversion efficiency of PPD to ginsenoside CK. We used a mixture of ethanol and glycerol to enable a CK production of $1.70 \pm 0.16 \mathrm{~g} / \mathrm{L}$. To the best of our knowledge, this is the highest reported levels of CK production.

\section{Results and discussion}

\section{Construction of strains producing $\mathrm{CK}$}

In order to obtain a strain producing ginsenoside CK, the previously constructed PPD producing $S$. cerevisiae WLT-MVA5 [26] was used as a parent strain for further studies. First, we overexpressed the UGT1 gene encoding glycosyltransferase that catalyzes the synthesis of CK from PPD. The UGT1 gene expression cassette was inserted into the ade 2 site of the WLT-MVA5, and the strain WLN-1 was obtained. The CK production of WLN-1 at $96 \mathrm{~h}$ was $146.79 \pm 5.11 \mathrm{mg} / \mathrm{L}$, and the PPD production was $370.24 \pm 10.23 \mathrm{mg} / \mathrm{L}$ (Fig. 2). The conversion rate from PPD to ginsenoside CK was $22.68 \pm 0.29 \%$. The conversion rate was low, and $77.32 \%$ of PPD remained unconverted. In order to promote the transformation of PPD, we tried increasing the UDPG supply. The gene UGP1 encoding UTP-glucose-1-phosphate uridylyltransferase 1 which catalyzes the synthesis of UDPG from glucose-1-phosphate was overexpressed, and the strain WLN-2 was obtained. The CK production by WLN-2 reached $211.42 \pm 11.01 \mathrm{mg} / \mathrm{L}$ at $96 \mathrm{~h}$, the PPD production was $206.39 \pm 6.32 \mathrm{mg} / \mathrm{L}$, and the conversion rate was $43.11 \pm 0.54 \%$. In order to further increase the production of CK, we overexpressed the gene PGM2 encoding phosphoglucomutase 2, which catalyzes the synthesis from glucose-6-phosphate to glucose-1-phosphate, and PGK1p-PGM2-PGM2t, TDH3P-UGP1-UGP1t, and TEF1p-UGT1-ADH1t expression cassettes were inserted into the single copy site ade2 of $S$. cerevisiae WLTMVA5 to obtain the strain WLN-3. The CK production of WLN-3 reached $263.94 \pm 2.36 \mathrm{mg} / \mathrm{L}$ at $96 \mathrm{~h}$, the PPD production was $108.75 \pm 2.90 \mathrm{mg} / \mathrm{L}$, and the conversion rate was $64.23 \pm 0.4 \%$. After overexpression of UDPG synthesis-related genes, the production of CK increased by $79.81 \%$, and the conversion rate increased by $183.20 \%$. As depicted in Fig. 2, CK production was limited by the 


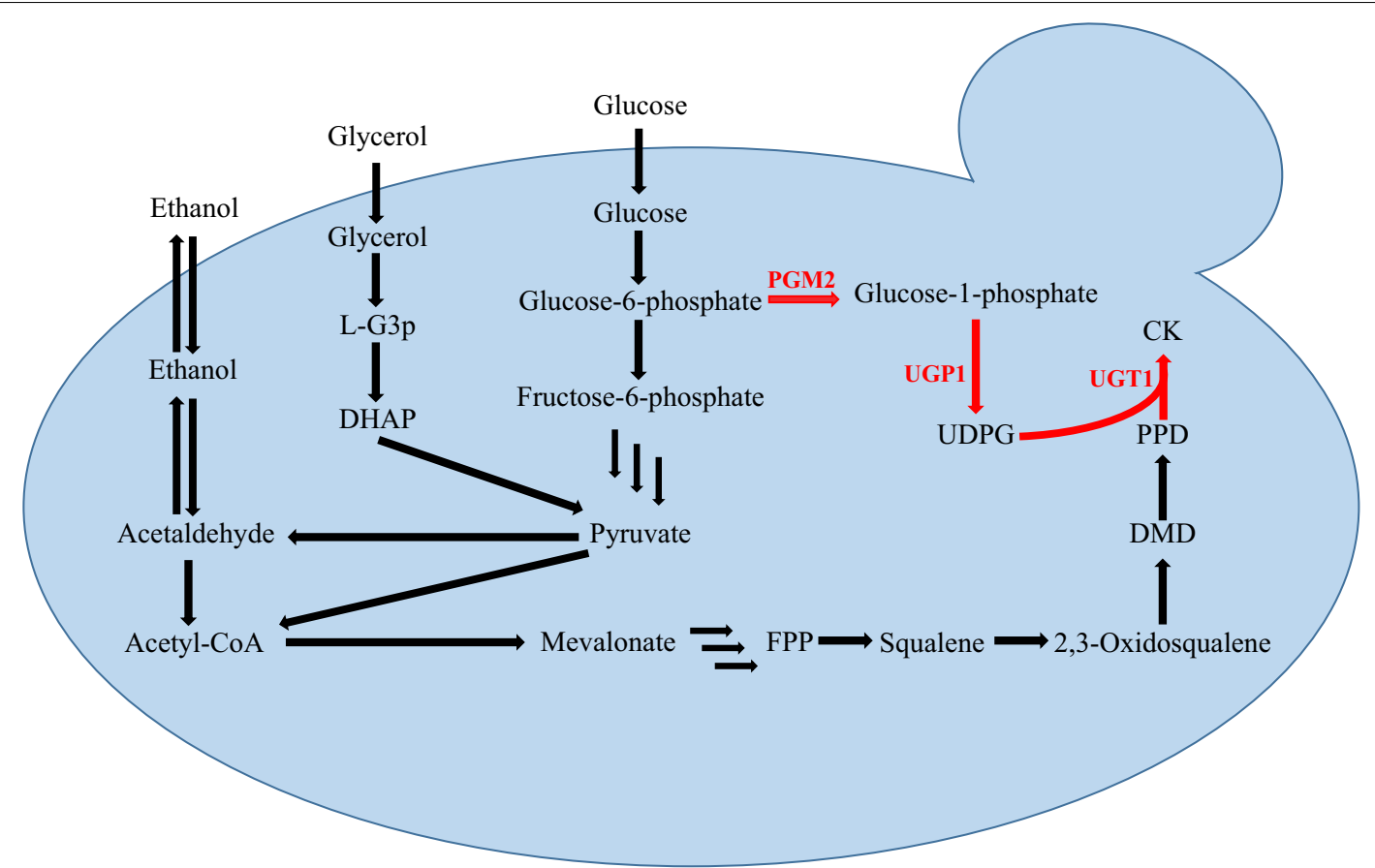

Fig. 1 CK synthesis pathway in Saccharomyces cerevisiae. PGM2 phosphoglucomutase 2, UGP1 UTP-glucose-1-phosphate uridylyltransferase 1, UGT1 UDP-glucose glucosyltransferase, FPP farnesyl diphosphate, DMD dammarenediol-II, PPD protopanaxadiol, CK compound K, UDPG uridine diphosphate-glucose

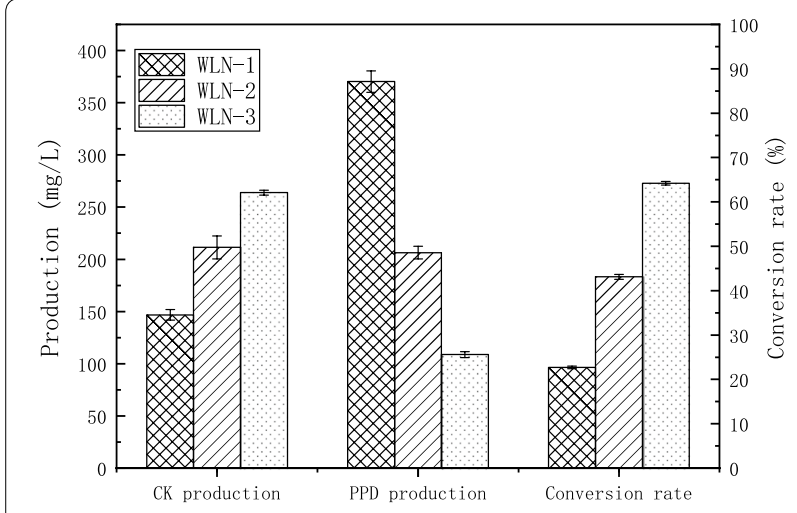

Fig. 2 Comparison of fermentation by WLN-1, WLN-2, and WLN-3 strains. WLN-1, WLN-2, and WLN-3 were fermented in YPD medium for $96 \mathrm{~h}$

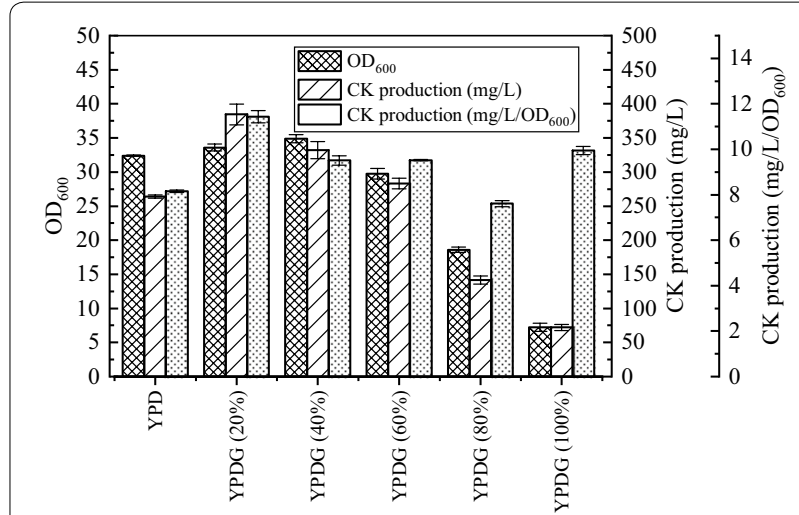

Fig. 3 Effect of different glycerol to glucose ratios on CK production. YPDG (20\%), YPDG (40\%), YPDG (60\%), YPDG (80\%), YPDG (100\%), respectively represent $20,40,60,80$, and $100 \%$ glucose in YPD replaced by glycerol of equimolar carbon quantity supply of UDPG and increased by $79.81 \%$ after PGM2 and UGP1 overexpression compared to that in WLN-1.

\section{Effect exerted by glycerol as partial carbon source on CK production}

Dutra et al. observed that glycerol increases UDPG pyrophosphatase activity relative to glucose [24]. We prepared a series of fermentation mediums using glucose and glycerol as carbon sources, that had the same molar concentration of carbon as YPD, and compared the effects of the medium with different glycerol to glucose ratios (see materials and methods) on CK production by WLN-3. The data is presented in Fig. 3. Based on Fig. 3, YPDG (20\%) exhibited the highest CK production, YPDG $(40 \%)$ exhibited the highest $\mathrm{OD}_{600}$. However, $\mathrm{OD}_{600}$ 
reduces with the increase in the proportion of glycerol (above 40\%). YPDG (20\%) is exhibits the highest increase in unit $\mathrm{OD}_{600}$, and apart from for YPDG (80\%), the other media containing glycerol exhibit a higher increase in unit $\mathrm{OD}_{600}$ than YPD. Wilson et al. observed that when S. cerevisiae is cultured in glycerol, the levels of glycogen accumulated are greater than that when cultured in glucose, and the precursor of glycogen synthesis is UDPG [25]. Glycerol promotes CK production possibly because it increases the supply of UDPG or the activity of glycosyltransferases. Glycerol promotes cell growth possibly since (1) it is more reduced than glucose [20], (2) it improves the stability of cell membrane proteins [22], and (3) it promotes the correct folding of nascent polypeptides [23]. The $\mathrm{OD}_{600}$ of yeast cultured with pure glycerol as carbon source is 7.25 , indicating that glycerol utilization is relatively low and inhibits yeast growth. Therefore, with reduction in glucose concentration and increase in glycerol concentration, $\mathrm{OD}_{600}$ increased at first and then decreased. With increase in glycerol concentration (above 20\%), the yield of CK decreased significantly. When the glycerol concentration was above $40 \%$, yeast growth was inhibited. When the glycerol concentration is $80 \%$, the effect exerted by glycerol on CK production is stronger than the effect exerted on growth, and hence increase in unit $\mathrm{OD}_{600}$ is lower than that in the control; when glycerol concentration is $100 \%$, the inhibitory effect of glycerol on cell growth exceeds the effect on CK production, and hence it is higher than that observed in the control. The presence of glycerol favors the synthesis of CK compared to presence of glucose in isolation, although the growth of the strain is dependent on glucose.
From Fig. 3, it can be inferred that YPDG (20\%) is most favorable for ginsenoside $\mathrm{CK}$ synthesis; hence, the fermentation in the medium with YPD and YPDG (20\%) is monitored and $\mathrm{OD}_{254}$ is used to represent the relative concentration of UDPG (Fig. 4). Yeast utilizest glucose and produces ethanol simultaneously. After the depletion of glucose, the yeast begins to consume ethanol when YPD medium is used. However, ethanol and glycerol were co-consumed when the strain was cultivated in YPDG (20\%) medium. At $36 \mathrm{~h}$, ethanol was exhausted in both media, and glycerol consumption ceased in YPDG (20\%). The production of PPD and CK in the two media had no significant difference before $36 \mathrm{~h}$. However, the accumulation of UDPG in YPDG (20\%) was higher than that in YPD, indicating that the presence of glycerol could promote UDPG accumulation. After $36 \mathrm{~h}$, CK production of YPDG (20\%) was higher than that of YPD, and the conversion rate of PPD to CK in YPDG $(20 \%)$ was higher than that in YPD. Lastly, in YPD, the production of CK was $263.94 \pm 2.36 \mathrm{mg} / \mathrm{L}$, the conversion rate was $64.23 \pm 0.41 \%$, and the yield from glucose was $10.56 \pm 0.09 \mathrm{mg} / \mathrm{g}$. In YPDG (20\%), the production of CK was $384.52 \pm 15.23 \mathrm{mg} / \mathrm{L}, 45.68 \%$ higher than that in YPD, the conversion rate was $77.37 \pm 3.37 \%, 13.14 \%$ higher than that in YPD, the yield from carbon (glucose and glycerol) was $15.60 \pm 0.62 \mathrm{mg} / \mathrm{g}, 47.73 \%$ higher than that in YPD, and the total concentration of PPD and CK was $25.86 \%$ higher than that in YPD. The overall levels of UDPG in YPDG (20\%) was marginally higher than that in YPD. The addition of glycerol promoted PPD glycosylation and induced greater carbon flow to ginsenosides synthesis.
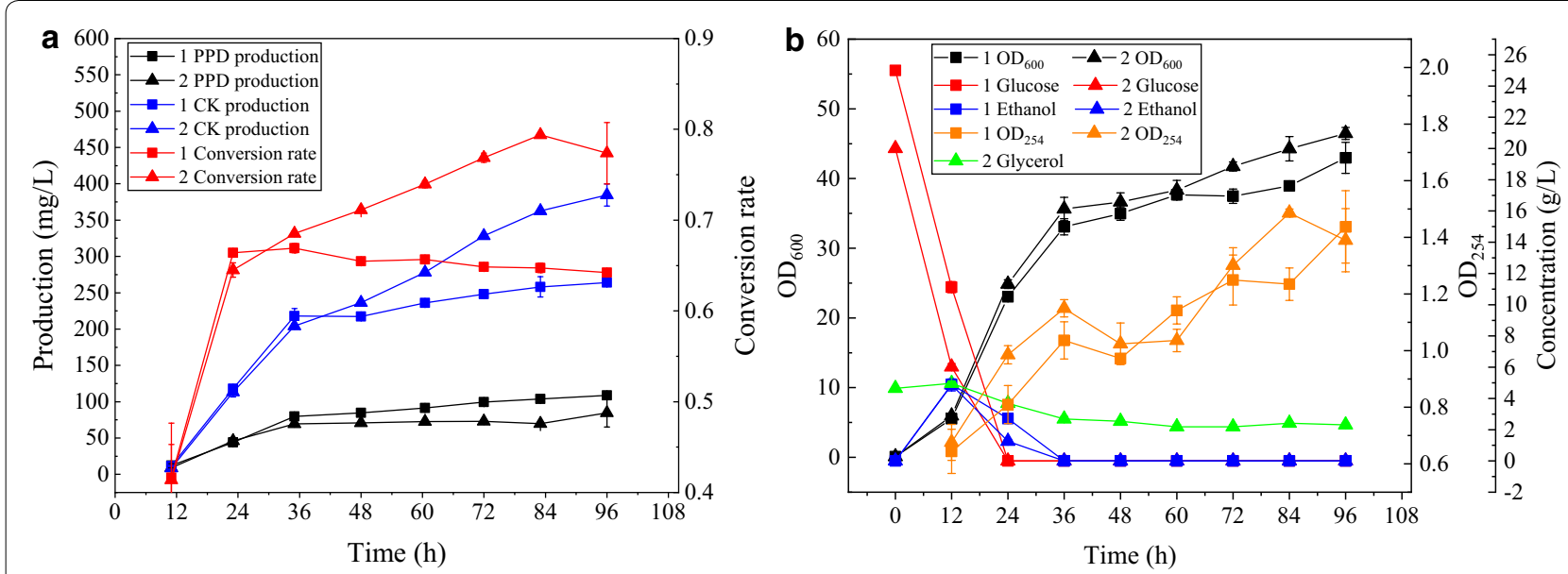

Fig. 4 Comparison of fermentation process between YPD and 20\% glycerol medium. a Alterations in production during fermentation. b Other parameters altered during fermentation. In the legend, 1 represents YPD, and 2 represents YPDG (20\%) (20\% of glucose in YPD is replaced by an equimolar carbon quantity of glycerol). $\mathrm{OD}_{254}$ represents the relative quantity of UDPG 


\section{Fermentation in the 5-L bioreactor}

For batch fermentation, YPDG (20\%) medium was used as the fermentation medium, and $10 \%$ inoculum was used. The fermentation data is presented in Fig. 5a. At $8 \mathrm{~h}$, glucose was depleted, while ethanol and glycerol consumption commenced; the ethanol concentration was lower than $1 \mathrm{~g} / \mathrm{L}$ at $16 \mathrm{~h}$, and the consumption rate of glycerol reduced. The production of $\mathrm{CK}$ at $96 \mathrm{~h}$ was $304.45 \pm 16.23 \mathrm{mg} / \mathrm{L}$, which was lower than that in the data from shake flask fermentation. On one hand, water volatilization in flask was more significant than that in the 5-L bioreactor, which inducing the concentration effect. On the other hand, the pool water solubility of CK made it adhere to the tank wall and stirring paddle during fermentation. In shake flask fermentation, the samples were collected by manual shaking. However, in the 5 -L bioreactor, ginsenosides on the tank wall and stirring paddle could not be collected during the fermentation process, and the concentration of these ginsenosides was calculated using the initial loading volume. Therefore, both biomass and product titer in batch fermenter were lower than those in the flask.

For fed-batch fermentation, as shown in Fig. 5a, it was confirmed that the fermentation medium contained $2.65 \mathrm{~g} / \mathrm{L}$ glycerol, $20 \mathrm{~g} / \mathrm{L}$ glucose, $20 \mathrm{~g} / \mathrm{L}$ peptone, and $10 \mathrm{~g} / \mathrm{L}$ yeast extract. When pure glycerol is used as a carbon source, cells exhibit poor growth. We have previously demonstrated that ethanol is more favorable for the synthesis of PPD than glucose [27]. In YPDG (20\%), ethanol and glycerol are simultaneously consumed after the glucose consumption, and the cells utilize glycerol negligibly after the ethanol is consumed. Since glycerol is beneficial to the formation of CK and ethanol is beneficial to the synthesis of PPD, we selected ethanol and glycerol for the feed. Based on the proportion and speed of consumption of ethanol and glycerol in batch fermentation, we commenced the feeding process at $16 \mathrm{~h}$, adding $15 \mathrm{~g}$ of ethanol and $6.6 \mathrm{~g}$ of glycerol every $8 \mathrm{~h}$; the fermentation data is presented in Fig. $5 \mathrm{~b}$. The final production levels achieved were $1.70 \pm 0.16 \mathrm{~g} / \mathrm{L}$.

\section{Conclusion}

We overexpressed the gene encoding the glycosyltransferase UGT1 and obtained a Saccharomyces cerevisiae strain that produced ginsenoside CK. Production was further increased by overexpression of UDPG supplyrelated genes. Our study indicated that glycerol can increase the conversion rate of PPD to CK. Fermentation conditions were optimized according to this feature. Lastly, the production levels in the 5 - $\mathrm{L}$ bioreactor reached $1.7 \mathrm{~g} / \mathrm{L}$. Use of $S$. cerevisiae in synthesis of glycosylated natural products has been reported widely $[16,28,29]$. The findings from this study may be used to synthesize other glycosylated natural products using S. cerevisiae, and the cost of production can be reduced by using glycerin to synthesize natural products. Recent studies have indicated that $S$. cerevisiae can efficiently synthesize ethanol from glycerol [30] and in the future study, this strategy can be used to further increase CK production in $S$. cerevisiae.
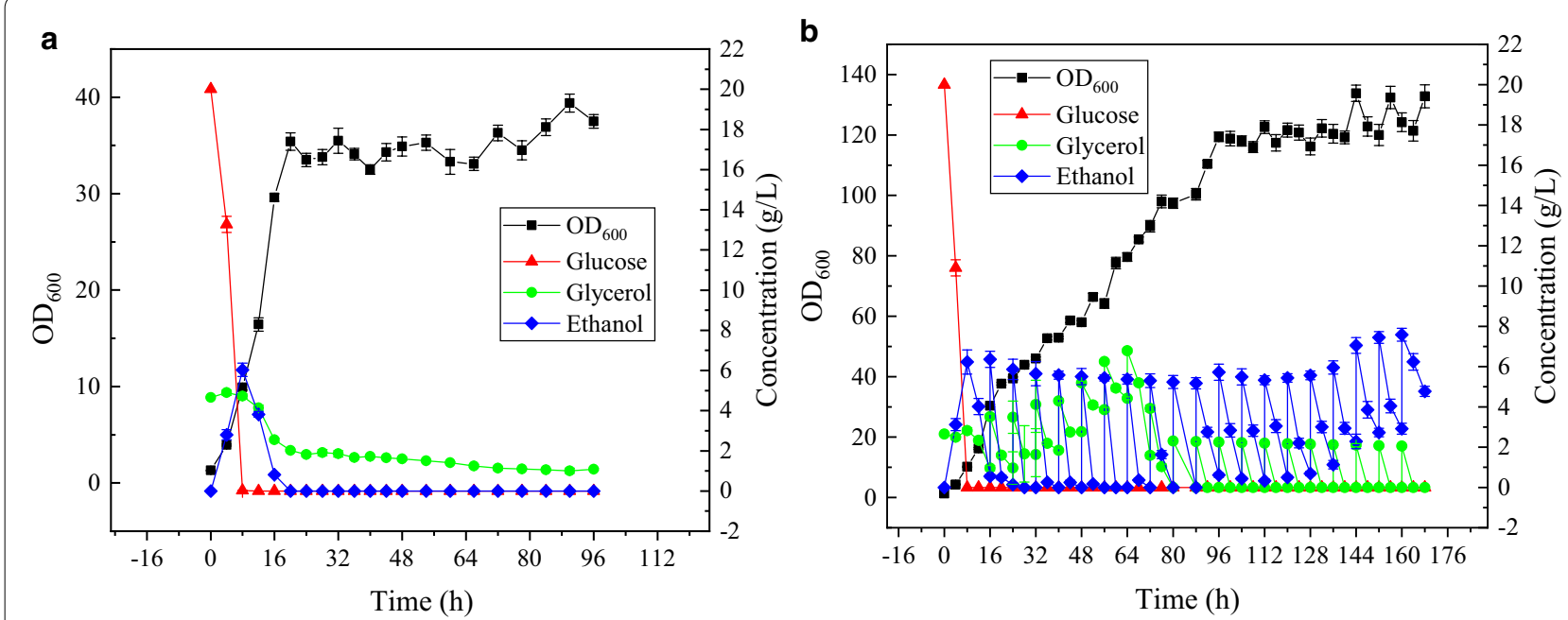

Fig. 5 Fermentation process in a $5-L$ bioreactor. a Batch fermentation. b Fed-batch fermentation 


\section{Materials and methods}

\section{Strains and medium}

The recently reported $S$. cerevisiae strain WLT-MVA5 was used as the parent strain [26]. The strain was stored at $-80{ }^{\circ} \mathrm{C}$ at $25 \%$ glycerol concentration. S. cerevisiae strains were cultured in YPD medium containing $25 \mathrm{~g} / \mathrm{L}$ glucose, $20 \mathrm{~g} / \mathrm{L}$ peptone, and $10 \mathrm{~g} / \mathrm{L}$ yeast extract. S. cerevisiae strain transformants were cultured in SD medium lacking adenine, tryptophan, leucine, uracil, and histidine where appropriate [27].

\section{Strain construction}

The UGT1 (GenBank: AIE12479.1) gene, encoding UDP-glycosyltransferase, was synthesized and cloned into pUC57 plasmids by GENEWIZ (Suzhou, China), with codon optimization for $S$. cerevisiae. The genome of $S$. cerevisiae BY4741 was used for PCR amplification of $A D E 2$ genes. The genome of $S$. cerevisiae W303-1a was used for PCR amplification of PGM2 and UGP1 genes. PGK1p-PGM2-PGM2t, TDH3p-UGP1-UGP1t, and TEF1p-UGT1-ADH1t expression cassettes were constructed by fusion PCR. The expression cassettes were integrated into the ade2 site of $S$. cerevisiae with selection marker ADE2.

\section{Flask fermentation}

The initial fermentation medium consisted of YPD medium containing $25 \mathrm{~g} / \mathrm{L}$ glucose, $20 \mathrm{~g} / \mathrm{L}$ peptone, and $10 \mathrm{~g} / \mathrm{L}$ yeast extract. A series of fermentation mediums using glucose and glycerol as carbon sources were prepared that, had the same molar concentration of carbon as YPD while they had different glucose to glycerol ratios. The carbon provided by glycerol accounted for $0,20,40,60,80$, and $100 \%$ of the total carbon provided by glycerol and glucose, respectively. Accordingly, these media were abbreviated as YPD, YPDG (20\%), YPDG (40\%), YPDG (60\%), YPDG (80\%), YPDG (100\%). The seeds for fermentation were cultured in YPD medium at $220 \mathrm{rpm}, 30{ }^{\circ} \mathrm{C}$ for $18 \mathrm{~h}$, and the conditions of flask fermentation were $220 \mathrm{rpm}$, fermentation at $30{ }^{\circ} \mathrm{C}$ for 4 days, and the initial inoculation OD was 0.1 .

\section{Fermentation in the 5-L bioreactor}

For batch fermentation in the 5-L bioreactor (Bailun, Shanghai, China), $200 \mathrm{~mL}$ of seed solution was cultured at $220 \mathrm{rpm}, 30{ }^{\circ} \mathrm{C}$ for $18 \mathrm{~h}$ and inoculated into $2 \mathrm{~L}$ YPDG (20\%) medium (20\% of glucose in YPD replaced by equal molar glycerol) at a fermentation temperature of $30{ }^{\circ} \mathrm{C}$ and an air flow rate of $2 \mathrm{~L} / \mathrm{min}$. The dissolved oxygen (DO) is maintained at $40 \%$ or more by adjusting the stirring speed. The $\mathrm{pH}$ was maintained at 5.5 by automated addition of $2 \mathrm{M}$ sulfuric acid and $5 \mathrm{M}$ aqueous ammonia.

For 5-L fed-batch fermentation, the fermentation medium contained $2.65 \mathrm{~g} / \mathrm{L}$ glycerol, $20 \mathrm{~g} / \mathrm{L}$ glucose, $20 \mathrm{~g} / \mathrm{L}$ peptone, and $10 \mathrm{~g} / \mathrm{L}$ yeast extract. At $16 \mathrm{~h}$ after start of fermentation, $350 \mathrm{~mL}$ of feed solution $(\mathrm{pH}=5.5)$ [26] and $25 \mathrm{~mL}$ of carbon source (glycerol $264 \mathrm{~g} / \mathrm{L}$, ethanol $600 \mathrm{~g} / \mathrm{L}$ ) were added. The carbon source was then added every $8 \mathrm{~h}$. Other conditions are identical to those for a single batch fermentation.

\section{Metabolite extraction and analysis}

Cell growth was determined by measuring the optical density at $600 \mathrm{~nm}\left(\mathrm{OD}_{600}\right)$ using a UV-VIS spectrophotometer. For the extraction and detection of PPD and CK, the fermentation broth was subjected to vortex agitation with n-butanol and 40-100 mesh $\mathrm{SiO}_{2}$. The mixture was then centrifuged, and the n-butanol phase was collected for HPLC analysis. HPLC analysis was conducted on Elite P230II high-pressure pump system equipped for UV detection at $203 \mathrm{~nm}$. Chromatographic separation was performed using the Hypersil C18 column $(4.6 \mathrm{~mm} \times 250 \mathrm{~mm}, 5 \mu \mathrm{m}$; Elite Analytical Instruments Co., Ltd., Dalian, China). Acetonitrile-water $(8: 2, \mathrm{v} / \mathrm{v})$ was used as the mobile phase. CK was determined by comparing the mass spectrogram with standards purchased from Meilun Biotechnology Co., Ltd (Dalian, China). Calculation of conversion rate from PPD to ginsenoside CK: PPD conversion rate $=\mathrm{M}_{\mathrm{CK}} /\left(\mathrm{M}_{\mathrm{CK}}+\mathrm{M}_{\mathrm{PPD}}\right)$. $\mathrm{M}_{\mathrm{CK}}$, molar concentration of $\mathrm{CK} ; \mathrm{M}_{\mathrm{PPD}}$, molar concentration of PPD.

UDPG measurements refer to the study of Daran et al. [31]. The fermentation broth was centrifuged to discard the supernatant, shaken, and boiling ethanol was added thereto, maintained at the specific temperature for 5-10 $\mathrm{min}$, and then freeze-dried. Three milliliter of $50 \mathrm{mM}$ Tris $(\mathrm{pH}=7.4)$ containing $1 \mathrm{mM}$ EDTA was added to the residue. The absorbance at $254 \mathrm{~nm}$ was measured using a spectrophotometer.

\section{Supplementary information}

Supplementary information accompanies this paper at https://doi. org/10.1186/s12934-020-01306-3.

Additional file 1: Table S1. Primers used for strains construction. Figure S1. LC/MS analysis of CK.

\section{Abbreviations}

PPD: Protopanaxadiol; CK: Compound K; UDPG: Uridine diphosphate-glucose; PGM2: Phosphoglucomutase 2; UGP1: UTP-glucose-1-phosphate uridylyltransferase 1; UGT1: UDP-glucose glucosyltransferase; FPP: Farnesyl diphosphate; DMD: Dammarenediol-II.

\section{Acknowledgements}

Not applicable. 


\section{Authors' contributions}

WHN designed the project, performed the main experiments and drafted the manuscript. FLZ and CBZ helped to design the experiment and drafted the manuscript; HYJ performed some experiments and collected the data; WYL supervised the project, analyzed the data and critically revised the manuscript. All authors read and approved the final manuscript.

\section{Funding}

This work was financially supported by the National Natural Science Foundation of China (No. 21878220)

\section{Availability of supporting data}

All data supporting the conclusions of this article are included within the manuscript and Additional file 1.

\section{Ethics approval and consent to participate}

Not applicable

\section{Consent for publication}

Not applicable.

\section{Competing interests}

The authors declare that they have no competing interests.

\section{Author details}

${ }^{1}$ School of Chemical Engineering and Technology, Tianjin University, Tianjin 300350, People's Republic of China. ${ }^{2}$ Key Laboratory of System Bioengineering (Tianjin University), Ministry of Education, Tianjin 300350, People's Republic of China. ${ }^{3}$ SynBio Research Platform, Collaborative Innovation Center of Chemical Science and Engineering (Tianjin), Tianjin 300350, People's Republic of China.

Received: 17 November 2019 Accepted: 10 February 2020

Published online: 19 February 2020

\section{References}

1. Leung KW, Wong AS-T. Pharmacology of ginsenosides: a literature review. Chin Med. 2010:5:20

2. Yang X-D, Yang Y-Y, Ouyang D-S, Yang G-P. A review of biotransformation and pharmacology of ginsenoside compound K. Fitoterapia. 2015;100:208-20.

3. Chen J, Wu H, Wang Q, Chang Y, Liu K, Wei W. Ginsenoside metabolite compound $\mathrm{K}$ suppresses T-cell priming via modulation of dendritic cell trafficking and costimulatory signals, resulting in alleviation of collageninduced arthritis. J Pharmacol Exp Ther. 2015;353:71.

4. Bae E-A, Park S-Y, Kim D-H. Constitutive $\beta$-gluccosidases hydrolyzing ginsenoside Rb1 and Rb2 from human intestinal bacteria. Biol Pharm Bull. 2000;23:1481-5.

5. Han BH, Park MH, Han YN, Woo LK, Sankawa U, Yahara S, Tanaka O. Degradation of ginseng saponins under mild acidic conditions. Planta Med. 1982;44:146-9.

6. Kim WY, Kim JM, Han SB, Lee SK, Kim ND, Park MK, Kim CK, Park JH. Steaming of ginseng at high temperature enhances biological activity. J Nat Prod. 2000;63:1702-4

7. Yan Q, Zhou X-W, Zhou W, Li X-W, Feng M-Q, Zhou P. Purification and properties of a novel beta-glucosidase, hydrolyzing ginsenoside Rb1 to CK, from Paecilomyces bainier. J Microbiol Biotechnol. 2008;18:1081-9.

8. Ro D-K, Paradise EM, Ouellet M, Fisher KJ, Newman KL, Ndungu JM, Ho KA, Eachus RA, Ham TS, Kirby J, et al. Production of the antimalarial drug precursor artemisinic acid in engineered yeast. Nature. 2006;440:940-3.

9. Zhang R, Cao Y, Liu W, Xian M, Liu H. Improving phloroglucinol tolerance and production in Escherichia coli by GroESL overexpression. Microb Cell Fact. 2017;16:227.

10. Kang M-K, Eom J-H, Kim Y, Um Y, Woo HM. Biosynthesis of pinene from glucose using metabolically-engineered Corynebacterium glutamicum. Biotechnol Lett. 2014:36:2069-77.

11. Yan X, Fan Y, Wei W, Wang P, Liu Q, Wei Y, Zhang L, Zhao G, Yue J, Zhou Z. Production of bioactive ginsenoside compound $\mathrm{K}$ in metabolically engineered yeast. Cell Res. 2014;24:770-3.
12. Gwak YS, Han JY, Adhikari PB, Ahn CH, Choi YE. Heterologous production of a ginsenoside saponin (compound $\mathrm{K}$ ) and its precursors in transgenic tobacco impairs the vegetative and reproductive growth. Planta. 2017;245:1105-19.

13. Li D, Wu Y, Zhang C, Sun J, Zhou Z, Lu W. Production of triterpene ginsenoside compound $\mathrm{K}$ in the non-conventional yeast Yarrowia lipolytica. J Agric Food Chem. 2019:67:2581-8.

14. Borodina I, Nielsen J. Advances in metabolic engineering of yeast Saccharomyces cerevisiae for production of chemicals. Biotechnol J. 2014:9:609-20.

15. Zhuang Y, Yang G-Y, Chen X, Liu Q, Zhang X, Deng Z, Feng Y. Biosynthesis of plant-derived ginsenoside $R h 2$ in yeast via repurposing a key promiscuous microbial enzyme. Metab Eng. 2017;42:25-32.

16. Wang H, Yang Y, Lin L, Zhou W, Liu M, Cheng K, Wang W. Engineering Saccharomyces cerevisiae with the deletion of endogenous glucosidases for the production of flavonoid glucosides. Microb Cell Fact. 2016;15:134.

17. Kumar P, Ray S, Patel SKS, Lee J-K, Kalia VC. Bioconversion of crude glycerol to polyhydroxyalkanoate by Bacillus thuringiensis under non-limiting nitrogen conditions. Int J Biol Macromol. 2015;78:9-16.

18. Tan HW, Abdul Aziz AR, Aroua MK. Glycerol production and its applications as a raw material: a review. Renew Sustain Energy Rev. 2013;27:118-27.

19. Kumar P, Mehariya S, Ray S, Mishra A, Kalia VC. Biodiesel industry waste: a potential source of bioenergy and biopolymers. Indian J Microbiol. 2015;55:1-7.

20. Maru BT, Bielen AAM, Kengen SWM, Constantí M, Medina F. Biohydrogen production from glycerol using Thermotoga spp. Energy Procedia. 2012;29:300-7.

21. Ochoa-Estopier A, Lesage J, Gorret N, Guillouet SE. Kinetic analysis of a Saccharomyces cerevisiae strain adapted for improved growth on glycerol: implications for the development of yeast bioprocesses on glycerol. Bioresour Technol. 2011;102:1521-7.

22. Gekko K, Timasheff SN. Mechanism of protein stabilization by glycerol: preferential hydration in glycerol-water mixtures. Biochemistry. 1981;20:4667-76.

23. Meng F-G, Park Y-D, Zhou H-M. Role of proline, glycerol, and heparin as protein folding aids during refolding of rabbit muscle creatine kinase. Int J Biochem Cell Biol. 2001:33:701-9.

24. Dutra MB, Silva JT, Mattos DC, Panek AD. Regulation of UDPG-pyrophosphorylase isoforms in Saccharomyces cerevisiae and their roles in trehalose metabolism. Biochim et Biophys Acta (BBA). 1996;1289:261-9.

25. Wilson WA, Hughes WE, Tomamichel W, Roach PJ. Increased glycogen storage in yeast results in less branched glycogen. Biochem Biophys Res Commun. 2004:320:416-23.

26. Zhao F, Bai P, Nan W, Li D, Zhang C, Lu C, Qi H, Lu W. A modular engineering strategy for high-level production of protopanaxadiol from ethanol by Saccharomyces cerevisiae. AlChE J. 2019;65:866-74

27. Zhao F, Du Y, Bai P, Liu J, Lu W, Yuan Y. Enhancing Saccharomyces cerevisiae reactive oxygen species and ethanol stress tolerance for high-level production of protopanoxadiol. Bioresour Technol. 2017;227:308-16.

28. Levisson M, Patinios C, Hein S, de Groot PA, Daran J-M, Hall RD, Martens S, Beekwilder J. Engineering de novo anthocyanin production in Saccharomyces cerevisiae. Microb Cell Fact. 2018;17:103.

29. Strucko T, Magdenoska O, Mortensen UH. Benchmarking two commonly used Saccharomyces cerevisiae strains for heterologous vanillin- $\beta$-glucoside production. Metab Eng Commun. 2015;2:99-108.

30. Aßkamp MR, Klein M, Nevoigt E. Saccharomyces cerevisiae exhibiting a modified route for uptake and catabolism of glycerol forms significant amounts of ethanol from this carbon source considered as 'non-fermentable'. Biotechnol Biofuels. 2019:12:257.

31. Daran JM, Dallies N, Thines-Sempoux D, Paquet V, François J. Genetic and biochemical characterization of the UGP1 gene encoding the UDPglucose pyrophosphorylase from Saccharomyces cerevisiae. Eur J Biochem. 1995:233:520-30.

\section{Publisher's Note}

Springer Nature remains neutral with regard to jurisdictional claims in published maps and institutional affiliations. 